УДК 621.314.57

\title{
СИНТЕЗ АЛГОРИТМА УПРАВЛЕНИЯ АВТОНОМНОЙ СИСТЕМОЙ ЭНЕРГОСНАБЖЕНИЯ С ИСПОЛЬЗОВАНИЕМ КВАТЕРНИОНОВ
}

\author{
Нос Олег Викторович1, \\ nos@corp.nstu.ru \\ Коровин Александр Владимирович2, \\ akvkorovin@ya.ru \\ Кучак Сергей Викторович', \\ kuchak.2012@corp.nstu.ru \\ 1 Новосибирский государственный технический университет, \\ Россия 630073, г. Новосибирск, пр. К. Маркса, 20. \\ $2 \mathrm{AO}$ «ПО «CЕВЕР», \\ Россия, 630027, г. Новосибирск, ул. Объединения, 3.
}

\begin{abstract}
Актуальность. В связи с непрерывным увеличением доли производства электрической энергии на основе возобновляемых источников, к числу которых относят солнечное излучение, энергия ветра, морские приливы и т. д., которые характеризуются непостоянством и случайным характером периодов генерации мощности, все большее значение приобретают прикладные задачи бесперебойного электропитания автономных объектов с гарантированным качеством и высоким быстродействием. Зачастую нагрузка также имеет непредсказуемый характер, что проявляется в виде несимметрии подключения, скачкообразных сбросов и набросов мощности, а также в наличии нелинейных потребителей, что отражается на качестве напряжения в системе электроснабжения.

Цель: синтез быстродействующего алгоритма управления автономной трехфазной системой электроснабжения технологических объектов минерально-сырьевого комплекса с целью обеспечения симметричного гармонического закона изменения во времени генерируемых напряжений.

Объекты: автономная система электропитания; четырехстоечный мостовой инвертор с широтно-импульсной модуляцией, односразная/трехфазная нагрузка.

Методы: алгебра кватернионов, некоммутативные правила умножения мнимых единиц, принцип управления по отклонению, системы подчиненного регулирования.

Результаты. Разработана базовая концепция построения, организации и технической реализации автономной системы генерации электрической энергии переменного тока, способ управления которой базируется на декомпозиции кватерниона трехфазных напряжений на симметричную гармоническую составляющую и компоненты, вызваннье амплитудно-фазовой асимметрией и нелинейностью цепей нагрузки. Описанный в работе непрерывный закон обеспечивает высокое быстродействие мгновенной компенсации искажений в режимах отработки скачкообразных сбросов и набросов нагрузки. Экспериментальные исследования проведены на нагрузке различного рода и конфигурации: однофазной, трехфазной симметричной, а также двухфазной, одна фраза из которых имеет нелинейный характер потребления тока.
\end{abstract}

\section{Ключевые слова:}

Автономная система электроснабжения, автономный инвертор напряжения, однофразная нагрузка, трехфазная нагрузка, кватернион напряжений.

\section{Введение}

В настоящее время в связи с интенсивным освоением природных ресурсов в районах Крайнего Севера и Арктического региона, относящихся к неэлектрифицированным территориям, все большую актуальность и значимость приобретают прикладные задачи бесперебойного и качественного электропитания основного и вспомогательного технологического оборудования, занятого в процессе добычи, подготовки и переработки полезных ископаемых [1]. Автономные системы электроснабжения данного класса, как правило, состоят из одной или нескольких дизельгенераторных электростанций соответствующей мощности с ветро-солнечными установками, аккумуляторной батареи, промежуточного накопителя энергии емкостного типа, а также автономного инвертора напряжения на основе трёхстоечной топологии с разделительными конденсаторами [2] или четырёхстоеч- ной мостовой схемы [3] с выходным индуктивноемкостным (LC) фильтром, причем последнее схемотехническое решение обладает рядом преимуществ над первым [4].

Отличительной особенностью эксплуатации электротехнического комплекса данного типа является наличие существенно нелинейных трехфазных промышленных и, в ряде случаев, однофазных коммунально-бытовых потребителей. Такие нагрузки вызывают протекание большого тока в нулевом проводе, а также амплитудно-фазовую асимметрию и искажения в мгновенной форме генерируемых напряжений, которые приводят к дополнительным среднеквадратичным потерям в распределительной линии в совокупности с интенсивным старением изоляции [5]. Для исключения негативного влияния вышеуказанных возмущающих факторов на практике применяют специальные алгоритмы компенсации на основе класси- 
ческих пропорционально-интегральных [6], пропорционально-резонансных [7] и пропорциональноинтегрально-дифференцирующих [8] регуляторов в различных системах координат [9], нелинейных гистерезисных законах [10], скользящих режимах [11], модального [12] или прогнозирующего [13] управления и т. д., которые, однако, не обеспечивают быструю реакцию на скачкообразный сброс/наброс нагрузки и приемлемое качество электрической энергии. В этой связи данная статья посвящена новому подходу к синтезу быстродействующей системы управления четырехстоечным полупроводниковым преобразователем в кватернионном базисе, позволяющему устранить из состава генерируемых трехфазных напряжений обратную и нулевую последовательность фаз на основной частоте, а также высокочастотные гармонические составляющие.

\section{Математическая модель}

\section{четырехпроводной системы генерирования}

На рис. 1 изображена упрощенная функциональная схема автономной системы электроснабжения, состоящей из инвертора напряжений АИН с полностью управляемыми полупроводниковыми ключами, собранного по мостовой схеме с четырьмя стойками, звена постоянного тока с напряжением $u_{d c}$, формируемого при помощи внешнего источника электрической энергии, силового LC-фильтра с емкостью конденсаторов $C_{f}$ и тремя одинаковыми дросселями с активным сопротивлением $R_{f}$ и индуктивностью $L_{f}$, а также дросселя с параметрами $R_{N}, L_{N}$, включенного между четвертой стойкой $N$ и нейтральной точкой $O$ нагрузки произвольного вида [14].

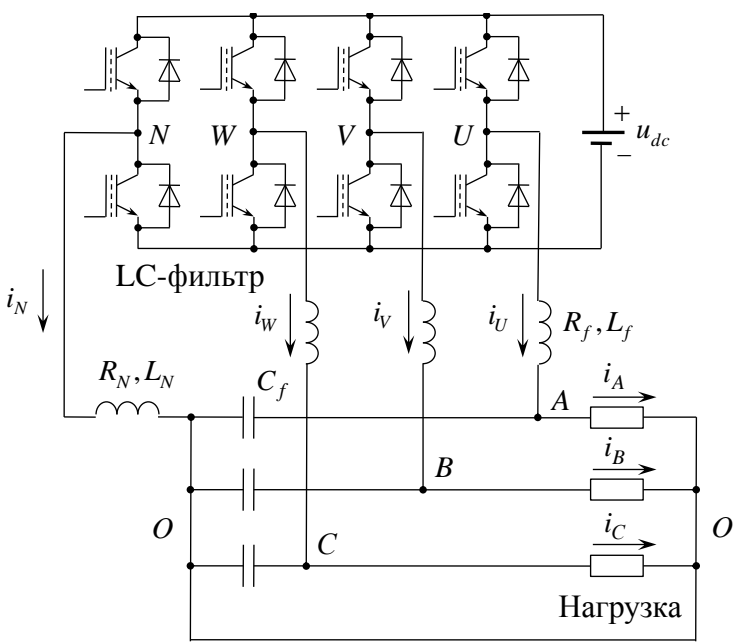

Puc. 1. Упрощенная функииональная схема трехфазной системы электроснабжения на базе четырехстоечного мостового инвертора

Fig. 1. Simplified configuration of three-phase distribution generation system based on four-leg full-bridge inverter

Векторно-матричная математическая модель силовой схемы, изображенной на рис. 1, описывается следующими дифференциальными и алгебраическими уравнениями:

$$
\left\{\begin{array}{c}
L_{f} \frac{d \mathbf{i}_{U W V}}{d t}=u_{d c} \mathbf{S}-R_{f} \mathbf{i}_{U W V}-\mathbf{u}_{A B C}+L_{N} \frac{d \mathbf{i}_{N}}{d t}+R_{N} \mathbf{i}_{N}, \\
C_{f} \frac{d \mathbf{u}_{A B C}}{d t}=\mathbf{i}_{U W V}-\mathbf{i}_{A B C}, \\
i_{N}=-\sum_{j=U, V, W} i_{j},
\end{array}\right.
$$

где $\mathbf{u}_{A B C}$ - алгебраический вектор трехфазных напряжений на выходе LC-фильтра

$$
\mathbf{u}_{A B C}=\left[\begin{array}{lll}
u_{A O} & u_{B O} & u_{C O}
\end{array}\right]^{\mathrm{T}} ;
$$

$\mathbf{i}_{U V W}, \mathbf{i}_{A B C}, \mathbf{i}_{N}$ - алгебраические векторы токов на выходе АИН, в нагрузке и в дросселе с параметрами $R_{N}, L_{N}$ соответственно

$$
\begin{gathered}
\mathbf{i}_{U V W}=\left[\begin{array}{lll}
i_{U} & i_{V} & i_{W}
\end{array}\right]^{\mathrm{T}}, \mathbf{i}_{A B C}=\left[\begin{array}{lll}
i_{A} & i_{B} & i_{C}
\end{array}\right]^{\mathrm{T}}, \\
\mathbf{i}_{N}=\left[\begin{array}{lll}
i_{N} & i_{N} & i_{N}
\end{array}\right]^{\mathrm{T}} ;
\end{gathered}
$$

$\mathbf{S}$ - алгебраический вектор переключающих функций инвертора $S_{U}, S_{V}, S_{W}, S_{N}[13]$, которые принимают значение 1 при включении верхнего полупроводникового ключа соответствующей стойки и 0 при нахождении в открытом состоянии нижнего транзистора

$$
\mathbf{S}=\left[\begin{array}{c}
s_{U}-s_{N} \\
s_{V}-s_{N} \\
s_{W}-s_{N}
\end{array}\right] .
$$

В заключение также необходимо отметить, что математическая модель объекта в координатных осях $d, q, o$ представлена в [8].

\section{Декомпозиция кватерниона напряжения}

Математический аппарат кватернионов представляет собой некоммутативную алгебру с делением, оперирующую математическими объектами следующего вида $[15,16]$

$$
\boldsymbol{\Lambda}=\lambda_{0} 1+\lambda_{1} \mathbf{q}_{1}+\lambda_{2} \mathbf{q}_{2}+\lambda_{3} \mathbf{q}_{3},
$$

где $\lambda_{0}, \lambda_{1}, \lambda_{2}, \lambda_{3}$ - вещественные коэффициенты; $\mathbf{q}_{1}, \mathbf{q}_{2}$, $\mathbf{q}_{3}$ - мнимые единицы, для которых совместно с одной действительной 1 постулируются 16 правил умножения [17]

$$
1 \mathbf{q}_{k}=\mathbf{q}_{k} 1=\mathbf{q}_{k}, \quad \mathbf{q}_{k} \mathbf{q}_{l}=-\delta_{k l}+\varepsilon_{k l m} \mathbf{q}_{m},
$$

здесь $k, l, m=1,2,3$ - нижний индекс, показывающий порядковый номер мнимой единицы; $\delta_{k l}$ - трехмерный символ Кронекера, симметричный по своим индексам $\delta_{k l}=\delta_{l k}$

$$
\delta_{k l}=\left\{\begin{array}{l}
1, \text { при } k=l, \\
0, \text { при } k \neq l ;
\end{array}\right.
$$

$\varepsilon_{k l m}$ - трехмерный символ Леви-Чивиты, антисимметричный по индексам, равный

$$
\varepsilon_{123}=\varepsilon_{231}=\varepsilon_{312}=1, \varepsilon_{213}=\varepsilon_{321}=\varepsilon_{132}=-1
$$

и равный нулю, если два или три индекса равны друг другу. 
Первый член в правой части (1) носит название скалярной (действительной) части $\operatorname{scal} \Lambda=\lambda_{0}$, а три оставшиеся образуют между собой векторную (мнимую) составляющую

$$
\operatorname{vect} \mathbf{\Lambda}=\lambda_{1} \mathbf{q}_{1}+\lambda_{2} \mathbf{q}_{2}+\lambda_{3} \mathbf{q}_{3} .
$$

В свою очередь деление выполняется при помощи обратного кватерниона

$$
\boldsymbol{\Lambda}^{-1}=|\boldsymbol{\Lambda}|^{-2} \hat{\Lambda},
$$

здесь $\widehat{\boldsymbol{\Lambda}}=\operatorname{scal} \boldsymbol{\Lambda}-\operatorname{vect} \boldsymbol{\Lambda}-$ сопряженный кватернион; $|\boldsymbol{\Lambda}|=\sqrt{\boldsymbol{\Lambda} \widehat{\Lambda}}=\sqrt{\widehat{\Lambda} \Lambda}-$ модуль $\Lambda$.

На основании вышесказанного представим эталонные трехфазные гармонические напряжения с амплитудой $u_{m}=$ const и угловой частотой $\omega=100 \pi \mathrm{c}^{-1}$ следующего вида [5]

$$
\left\{\begin{array}{l}
u_{A O}^{*}=u_{m} \cos \omega t, \\
u_{B O}^{*}=u_{m} \cos (\omega t-2 \pi / 3), \\
u_{C O}^{*}=u_{m} \cos (\omega t+2 \pi / 3),
\end{array}\right.
$$

в форме чисто векторного кватерниона

$$
\mathbf{U}_{*}=u_{A O}^{*} \mathbf{q}_{1}+u_{B O}^{*} \mathbf{q}_{2}+u_{C O}^{*} \mathbf{q}_{3} .
$$

Для текущих значений переменных, формируемых на выходе силового LC-фильтра, по аналогии получим:

$$
\mathbf{U}_{A B C}=u_{A O} \mathbf{q}_{1}+u_{B O} \mathbf{q}_{2}+u_{C O} \mathbf{q}_{3} .
$$

Далее осуществим произведение двух данных кватернионов друг на друга, в результате чего с учетом (2) получаем

$$
\boldsymbol{\Delta}_{A B C}=\mathbf{U}_{*} \mathbf{U}_{A B C}=\operatorname{scal} \boldsymbol{\Delta}_{A B C}+\operatorname{vect} \boldsymbol{\Delta}_{A B C},
$$

здесь scal $\Lambda_{A B C}$, vect $\Lambda_{A B C}$ - скалярная и векторная компоненты специального кватерниона, который содержит в себе информацию обо всех мгновенных отклонениях между желаемыми $u_{A O}^{*}, u_{B O}^{*}, u^{*}$ СO и реальными $u_{A O}, u_{B O}, u_{C O}$ напряжениями

$$
\operatorname{scal} \Delta_{A B C}=\delta_{A B C}, \operatorname{vect} \boldsymbol{\Delta}_{A B C}=\delta_{A} \mathbf{q}_{1}+\delta_{B} \mathbf{q}_{2}+\delta_{C} \mathbf{q}_{3},
$$

здесь $\delta_{A B C}, \delta_{A}, \delta_{B}, \delta_{C}$ - вещественные коэффициенты вида

$$
\begin{gathered}
\delta_{A B C}=-u_{A O}^{*} u_{A O}-u_{B O}^{*} u_{B O}-u_{C O}^{*} u_{C O}, \\
\delta_{A}=u_{B O}^{*} u_{C O}-u_{C O}^{*} u_{B O}, \\
\delta_{B}=u_{C O}^{*} u_{A O}-u_{A O}^{*} u_{C O}, \delta_{C}=u_{A O}^{*} u_{B O}-u_{B O}^{*} u_{A O} .
\end{gathered}
$$

Основным достоинством данного подхода к анализу энергетических характеристик трехфазных систем переменного тока является возможность достаточно простым путем выделить из суммарного потока электрической энергии все неэффективные составляющие, вызванные асимметрией и нелинейностью процесса энергопотребления. В частности, как показано в $[17,18]$, в случае чисто резистивной симметричной трехфазной нагрузки scal $\Lambda_{A B C}=$ const и vect $\Lambda_{A B C}=0$, при наличии в электрической цепи реактивных элементов scal $\Lambda_{A B C}$ и vect $\Lambda_{A B C}$ становятся по- стоянными величинами, а при появлении искажений в мгновенной форме $u_{A O}, u_{B O}, u_{C O}$ в скалярной scal $\Lambda_{A B C}$ и векторной vect $\Lambda_{A B C}$ частях возникают мультигармонические колебания.

Для декомпозиции $\mathbf{U}_{A B C}$ воспользуемся обратным кватернионом эталонных напряжений

$$
\mathbf{U}_{A B C}=\mathbf{U}_{*}^{-1}\left(\operatorname{scal} \Delta_{A B C}+\operatorname{vect} \Delta_{A B C}\right)=\mathbf{U}_{A B C}^{+}+\mathbf{U}_{A B C}^{-},
$$
где $\mathbf{U}^{+}{ }_{A B C}, \mathbf{U}_{A B C}^{-}-$кватернионы, отвечающие за симметричную гармоническую составляющую прямой последовательности фаз и отклонения от $\mathbf{U}_{*}$ вследствие параметрической асимметрии и наличия высокочастотных гармоник.

$$
\begin{gathered}
\mathbf{U}_{A B C}^{+}=\mathbf{U}_{*}^{-1} \mathrm{scal} \overline{\boldsymbol{\Delta}}_{A B C}, \\
\mathbf{U}_{A B C}^{-}=\mathbf{U}_{*}^{-1}\left(\operatorname{scal} \tilde{\boldsymbol{\Delta}}_{A B C}+\operatorname{vect} \boldsymbol{\Delta}_{A B C}\right)= \\
=\mathbf{U}_{A B C}-\mathbf{U}_{*}^{-1} \mathrm{scal} \overline{\boldsymbol{\Delta}}_{A B C},
\end{gathered}
$$

здесь $\operatorname{scal} \overline{\boldsymbol{\Delta}}_{A B C}, \mathrm{scal} \tilde{\boldsymbol{\Delta}}_{A B C}-$ постоянная (средняя) и изменяющаяся во времени составляющие скалярной части $\Delta_{A B C}$ соответственно, причем [17]

$$
\text { scal } \mathbf{U}_{A B C}^{-}=\left|\mathbf{U}_{A B C}\right|^{-2}\left(u_{A O}^{*} \delta_{A}+u_{B O}^{*} \delta_{B}+u_{C O}^{*} \delta_{C}\right)=0 .
$$

В заключение также необходимо отметить, что применение кватернионов позволяет достаточно просто реализовывать координатное преобразование трехфазных переменных в силу того, что для данных целей используется только четыре кинематических параметра с одним уравнением связи вместо девяти элементов квадратной матрицы направляющих косинусов [15]. Так, например, инвариантный относительно скалярного произведения переход в координаты $\alpha, \beta, o$ (Clarke Transformation) [19] осуществляется при помощи следующего равенства

$$
\mathbf{X}_{\alpha \beta o}=\boldsymbol{\Lambda}_{\alpha \beta o} \mathbf{X}_{A B C} \boldsymbol{\Lambda}_{\alpha \beta o}^{-1}=x_{\alpha} \mathbf{q}_{1}+x_{\beta} \mathbf{q}_{2}+x_{o} \mathbf{q}_{3},
$$

здесь $\Lambda_{\alpha \beta o}-$ нормированный кватернион с вещественными коэффициентами

$$
\lambda_{0}=0,8805, \lambda_{1}=0,3647, \lambda_{2}=-0,2798, \lambda_{3}=0,1159 \text {, }
$$

а поворот на электрический угол $\gamma$ плоскости с осями $\mathbf{q}_{1}$, $\mathbf{q}_{2}$ относительно третьей мнимой единицы $\mathbf{q}_{3}$, который соответствует преобразованию Парка-Горева (Park Transformation) [20], выполняется в следующем виде

$$
\boldsymbol{\Lambda}_{d q o}=\cos \frac{\gamma_{u}^{*}}{2}+\sin \frac{\gamma_{u}^{*}}{2} \mathbf{q}_{3},
$$

в результате чего становится справедливым

$$
\begin{gathered}
\mathbf{X}_{d q o}=\left(x_{\alpha} \cos \gamma_{u}^{*}-x_{\beta} \sin \gamma_{u}^{*}\right) \mathbf{q}_{1}+ \\
+\left(x_{\alpha} \sin \gamma_{u}^{*}+x_{\beta} \cos \gamma_{u}^{*}\right) \mathbf{q}_{2}+x_{o} \mathbf{q}_{3}=x_{d} \mathbf{q}_{1}+x_{q} \mathbf{q}_{2}+x_{o} \mathbf{q}_{3} .(6)
\end{gathered}
$$

В свою очередь обратное координатное преобразование из $d, q, o$ в $\alpha, \beta$, о и затем из $\alpha, \beta, o$ в $A, B, C$ строится при помощи изменения порядка чередования прямого и обратного нормированных кватернионов

$$
\begin{aligned}
& \mathbf{X}_{\alpha \beta o}=\Lambda_{d q o}^{-1} \mathbf{X}_{d q o} \boldsymbol{\Lambda}_{d q o}=x_{\alpha} \mathbf{q}_{1}+x_{\beta} \mathbf{q}_{2}+x_{o} \mathbf{q}_{3}, \\
& \mathbf{X}_{A B C}=\boldsymbol{\Lambda}_{\alpha \beta o}^{-1} \mathbf{X}_{\alpha \beta o} \boldsymbol{\Lambda}_{\alpha \beta o}=x_{A} \mathbf{q}_{1}+x_{B} \mathbf{q}_{2}+x_{C} \mathbf{q}_{3} .
\end{aligned}
$$




\section{Система стабилизации трехфазных напряжений на нагрузке с использованием кватернионов}

Автономная система электропитания силового электрооборудования, занятого в технологических процессах добычи, транспортировании и переработки углеводородного сырья и других полезных ископаемых, а также коммунально-бытовых потребителей, строится в кватернионом базисе в соответствии с принципами управления по отклонению, последовательной коррекции и подчиненного регулирования [15], что иллюстрирует рис. 2, на котором используются следующие обозначения: ПК1, ПК2 - преобразователи кватерниона трехфазных токов инвертора из $A, B, C$ в $d, q, o$ и обратно, построенные на основании формул (5)-(8) соответственно; $\mathrm{PH}, \mathrm{PH}_{d q o}-$ ПИ-регуляторы модуля $\left|\mathbf{U}_{d q o}\right|$ и отклонений $u_{A O}, u_{B o}, u_{C O}$ от эталона (3); $\mathrm{PT}_{d q o}-$ трехканальный ПИ-регулятор выходного тока АИН в осях $d, q, o$; ТА - тригонометрический анализатор, осуществляющий вычисление $\left|\mathbf{U}_{*}\right|$ и оценку углового положения кватерниона трехфазных эталонных напряжений $\hat{\gamma}_{u}^{*}$; ДПР - динамическая подсистема определения текущей величины модуля измеренного кватерниона напряжений $\left|\mathbf{U}_{d q o}\right|$ и его разделения в $d, q$, o осях на две составляющие $\mathbf{U}^{+}{ }_{A B C}$ и $\mathbf{U}^{-}{ }_{A B C}$ в соответствии с (4), в которой для получения информации о постоянной составляющей $\operatorname{scal} \overline{\boldsymbol{\Delta}}_{d q o}$ использовался фильтр низких частот второго порядка с передаточной функцией

$$
W(p)=\frac{1}{p^{2}+A \Omega p+\Omega^{2}},
$$

здесь $p$ - оператор дифференцирования; $A, \Omega$ - коэффициент формы и среднегеометрический корень соответственно.

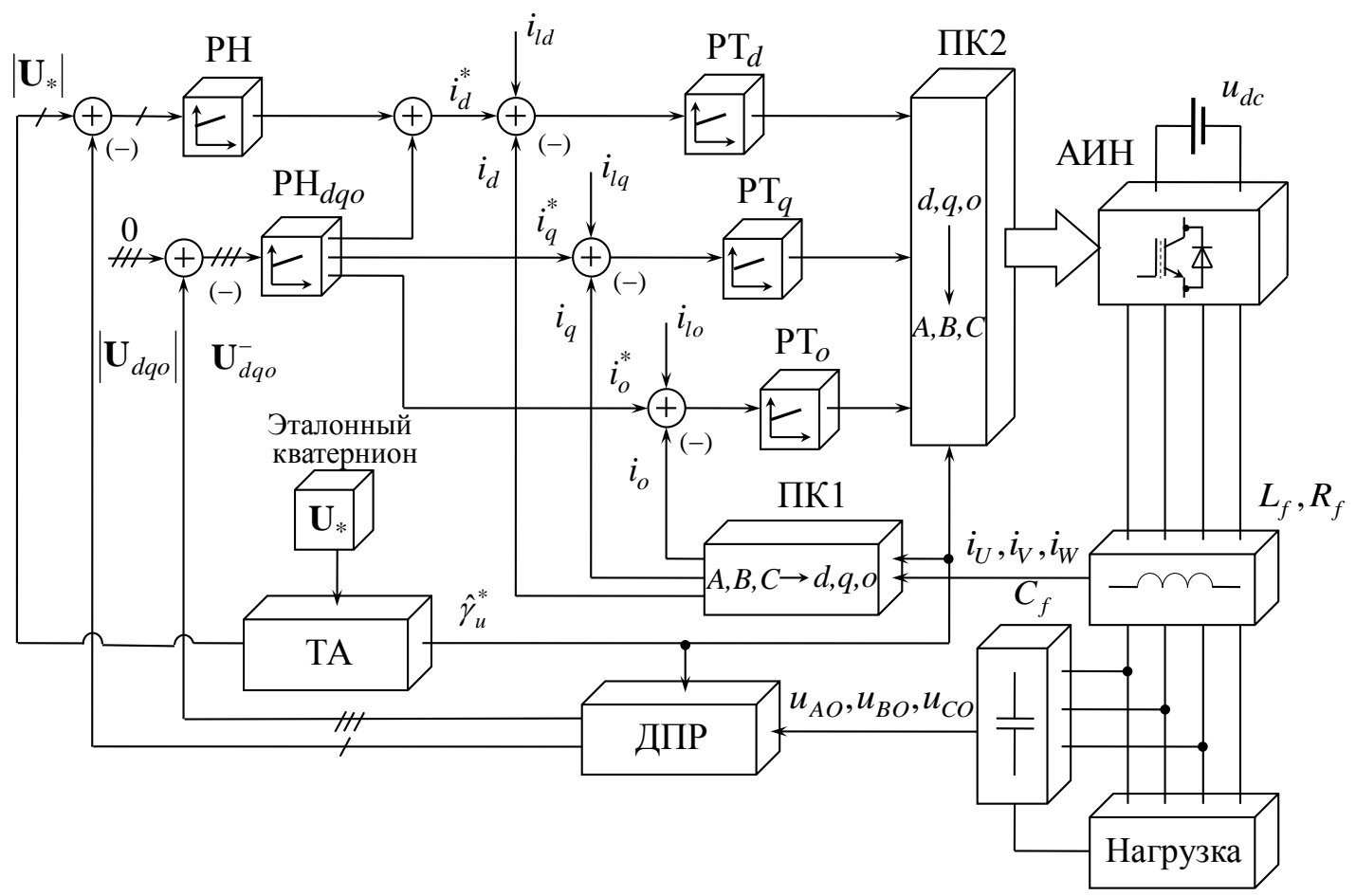

Pис. 2. Система управления четырехстоечным преобразователем в кватернионном базисе

Fig. 2. Control system of the four-leg VSI in quaternion domain

Для повышения быстродействия и компенсации внешних возмущений к выходам ПИ-регуляторов $\mathrm{PH}_{d q o}$ добавлялись токи нагрузки $i_{l d}, i_{l q}, i_{l o}$ в соответствующих осях после прохождения через апериодический фильтр с постоянной времени $\left(2 \pi 12 \cdot 10^{3}\right)^{-1}$ с [8].

В целях практической верификации разработанного алгоритма стабилизации напряжений на нагрузке в терминах алгебры кватернионов были выполнены экспериментальные исследования на специализированной установке, внешний вид которой приведен на рис. 3, которая состояла из полупроводникового преобразователя с частотой ШИМ, равной 15 кГц, и напряжением звена постоянного тока 539 В, силового LC-фильтра на выходе четырехстоечного мостового инвертора с параметрами $C_{f}=6,8$ мкФ и $L_{f}=0,58$ мГн, а также дросселя в нейтральной линии с аналогичной индуктивностью $L_{N}=0,58 \mathrm{м} \Gamma \mathrm{H}$.

Внутренний трехканальный контур регулирования выходных токов инвертора настраивался на полосу пропускания в замкнутом состоянии $\omega_{i}=2 \pi 750 \mathrm{c}^{-1}$, a внешний по отношению к нему контур модуля кватерниона текущих напряжений и их отклонений - на аналогичный параметр, равный $\omega_{u}=2 \pi 250 \mathrm{c}^{-1}$. Желаемое амплитудное значение напряжений $u_{m}$ принималось равным $250 \mathrm{~B}$, фильтр низких частот в блоке ДПР имел биномиальное распределение корней со среднегеометрическим корнем $100 \mathrm{c}^{-1}$, а фиксация трехфазных переменных осуществлялось с помощью многоканального измерительного комплекса «Genesis Gen7T». 


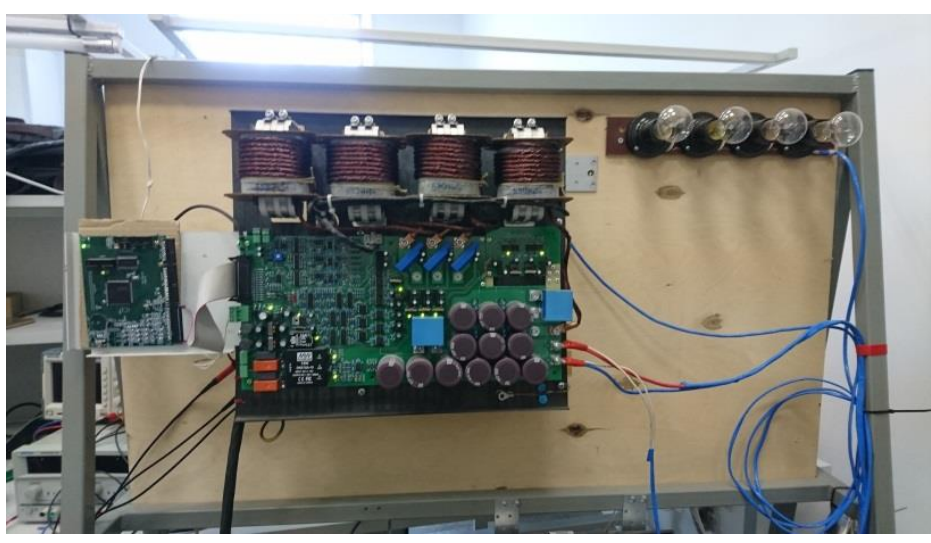

Рис. 3. Внешний вид экспериментальной установки

Fig. 3. Photograph of experimental platform

На рис. 4 изображен гармонический состав трехфазных напряжений на выходе силового LC-фильтра в режиме холостого хода, а на рис. $5, a, \sigma$ - осциллограммы напряжений и токов нагрузки при включении однофазного резистивного потребителя и скачкообразном уменьшении сопротивления двухфазной нелинейной цепи соответственно.

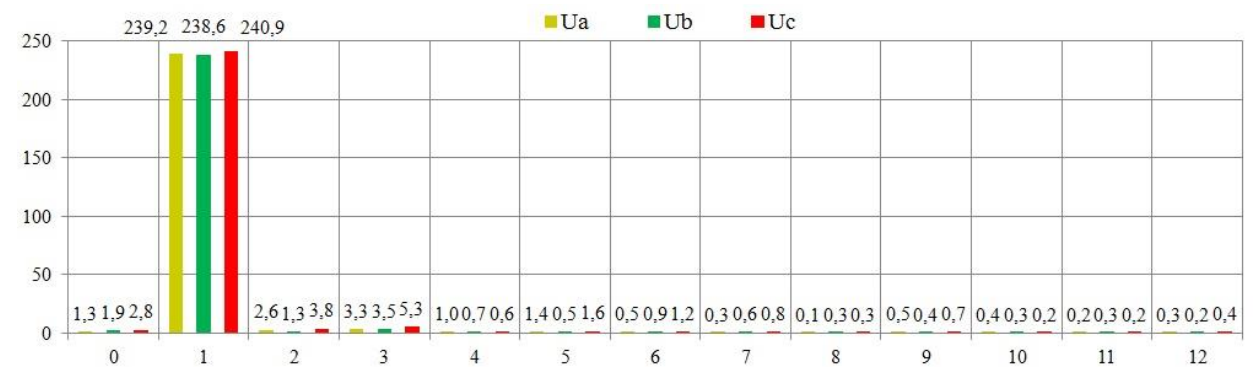

Pис. 4. Гармонический спектр генерируемых напряжений в режиме холостого хода

Fig. 4. Harmonic spectrum of produced voltages at no-load mode
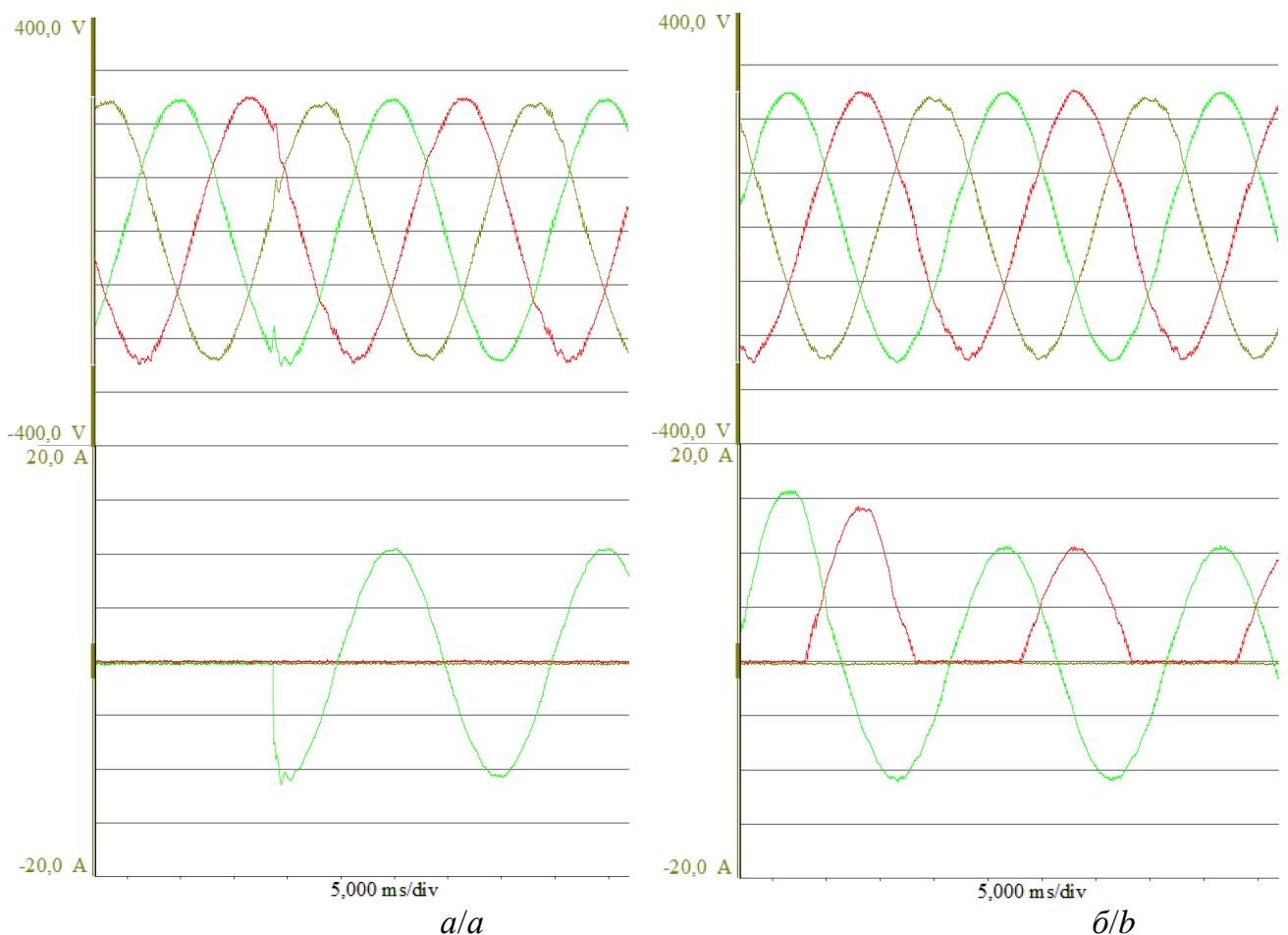

Pис. 5. Трехфазные напряжения и токи на стороне потребителя при скачкообразном изменении однофазной (a) и двухфазной (б) нагрузки

Fig. 5. Load three-phase voltages and currents under step change of single-phase (a), two-phase (b) loads 
Как видно из представленных выше результатов выполненного эксперимента, практическое применение алгебры кватернионов обеспечивает нормированное качество электрической энергии в статических режимах работы и требуемое быстродействие отработки внешних возмущающих воздействий в динамике. Генерируемые автономной системой электроснабжения трехфазные напряжения имеют близкую к гармонической мгновенную форму с незначительным отклонением установившегося амплитудного значения от заданной величины $u_{m}=250 \mathrm{~B}$.

\section{Заключение}

В данной статье описаны базовые положения нового алгоритмического подхода к построению и организации системы управления четырёхстоечным мостовым инвертором с выходным силовым LC-фильтром, предназначенным для бесперебойного питания различного рода технологического оборудования предприятий минерально-сырьевого комплекса, а также объектов аэрокосмической отрасли, основанного на использовании некоммута-

\section{СПИСОК ЛИТЕРАТУРЫ}

1. Выравнивание графика нагрузки предприятий за счет применения гибридных накопителей электроэнергии / O.С. Васильков, Д.Е. Батуева, К.А. Хомяков, П.С. Паляницин // Известия МГТУ «МАМИ». - 2020. - № 1. - С. 27-34.

2. Fraser M.E., Manning C.D., Wells B.M. Transformerless fourwire PWM rectifier and its application in AC-DC-AC converters // IEE Proceedings - Electric Power Applications. - 1995. - V. 142. № 6. - P. 410-416.

3. Jahns M., Maldonado M.A. A new resonant link aircraft power generating system // IEEE Transactions on Aerospace and Electronics Systems. - 1993. - V. 29. - № 1. - P. 206-214.

4. Vechiu I., Curea O., Camblong H. Transient operation of a four-leg inverter for autonomous applications with unbalanced load // IEEE Transactions on Power Electronics. - 2010. - V. 25. - № 2. - P. 399-407.

5. Nos O.V., Brovanov S.V., Dybko M.A. Development of active filtering algorithms for higher harmonics in electrical power circuits // Optoelectronics, Instrumentation and Data Processing. 2016. - V. 52. - № 6. - P. 557-562.

6. Hsu P., Behnke M. A three-phase synchronous frame controller for unbalanced load // 29th Annual IEEE Power Electronics Specialists Conference. - Fukuoka, Japan, 1998. - P. 1369-1374.

7. Rokrok E., Hamedani ME. Comprehensive control scheme for an inverterbased distributed generation unit // Iranian Journal of Science and Technology, Transaction B: Engineering. - 2009. - V. 33. - № B6. - P. 477-490.

8. Aboelsaud R., Ibrahim A., Garganeev A.G. Voltage control of autonomous power supply systems based on PID controller under unbalanced and nonlinear load conditions // International Youth Conference on Radio Electronics, Electrical and Power Engineering. - Moscow, 2019. - 6 p.

9. Aboelsaud R., Ibrahim A., Garganeev A.G. Review of three-phase inverters control for unbalanced load compensation // International Journal of Power Electronics and Drive System. - 2019. - V. 10. № 1. - P. 242-255

10. Prabhakar N., Mishra M. Dynamic hysteresis current control to minimize switching for three-phase four-leg VSI topology to compensate nonlinear load // IEEE Transactions on Power Electronics. - 2010. - V. 25. - № 8. - P. 1935-1942. тивной алгебры кватернионов. Описанный в работе непрерывный закон обеспечивает высокое быстродействие мгновенной компенсации искажений в режимах отработки скачкообразного сброса/наброса нагрузки, в том числе с нелинейными характеристиками, в совокупности с приемлемым гармоническим составом генерируемой электрической энергии и базируется на разделении кватерниона трехфазных напряжений на скалярную (действительную) и векторную (мнимую) части с последующим получением информации о положительной, отрицательной и нулевой последовательности фаз на основной частоте 50 Гц, а также о наличии мультигармонических составляющих. Математическое представление трехфазных переменных в форме кватернионов может быть применено к произвольной временной зависимости сигналов и не накладывает никаких ограничений на конкретную конфигурацию цепей потребителей, в качестве которых, например, могут выступать регулируемый электропривод переменного тока с преобразователем частоты или управляемый однофазный/трехфазный выпрямитель.

11. Zheng L., Le D. Control of a three-phase four-wire inverter // IECON 2012 - 38th Annual Conference on IEEE Industrial Electronics Society. - Montreal, QC, Canada, 2012. - P. 316-320.

12. Nasiri R., Radan A. Adaptive pole-placement control of 4-leg voltage-source inverters for standalone photovoltaic systems // Renewable Energy. - 2011. - V. 36 - № 7. - P. 2032-2042.

13. Digital predictive current control of a three-phase four-leg inverter/M. Rivera, V. Yaramasu, A. Llor, J. Rodriguez, B. Wu, M. Fadel // IEEE Transactions on Industrial Electronics. - 2013. - V. 60. - № 11. - P. 4903-4912.

14. Luis R., Silva J.F., Quadrado J.C. Quadrado output voltage quality evaluation of stand-alone four-leg inverters using linear and nonlinear controllers // Energies. - 2017. - V. 10. - № 4. - P. 1-20.

15. Quaternion control of four-leg inverter for distribution system with harmonic-producing load / O.V. Nos, A.V. Korovin, N.I. Nos, E.S. Kucher // The 11th International Conference on Electrical Power Drive Systems ICEPDS 2020. - Saint Petersburg, Russia, 2020. - 6 p.

16. Three-phase quaternion power in three-wire systems / A.S.F. Komeno, A.L.F. Filho, J.Y. Ishihara, V.P. Brasil // 19th International Conference on Renewable Energy and Power Quality Journal ICREPQ'21. - Almeria, Spain, 2021. - P. 493-498.

17. Nos O.V. Control strategy of shunt active power filter based on an algebraic approach // The 16th International Conference of Young Specialists on Micro/Nanotechnologies and Electron Devices EDM 2015. - Erlagol, Altai, Russia, 2015. - P. 459-463.

18. Nos O.V., Dudin A., Petzoldt J. The instantaneous power quaternion of the three-phase electric circuit with linear load // The 17th International Conference of Young Specialists on Micro/Nanotechnologies and Electron Devices EDM 2016. Erlagol, Altai, Russia, 2016. - P. 526-531.

19. Duesterhoeft W.C., Schulz M.W., Clarke E. Determination of instantaneous currents and voltages by means of alpha, beta, and zero components // Transactions of the American Institute of Electrical Engineers. - 1951. - V. 70. - № 2. - P. 1248-1255.

20. Park R.H. Two-reaction theory of synchronous machines // Transactions of the American Institute of Electrical Engineers. 1929. - V. 48. - № 3. - P. 716-727.

Поступила: 29.10 .20212$.

\section{Информация об авторах}

Hoc O.B., доктор технических наук, профессор кафедры проектирования технологических машин Новосибирского государственного технического университета.

Коровин A.B., заместитель главного конструктора по разработке новых изделий АО «ПО «СЕВЕР».

Кучак $\boldsymbol{C . B . , ~ к а н д и д а т ~ т е х н и ч е с к и х ~ н а у к , ~ с т а р ш и и ̆ ~ п р е п о д а в а т е л ь ~ к а ф е д р ы ~ э л е к т р о н и к и ~ и ~ э л е к т р о т е х н и к и ~ Н о - ~}$ восибирского государственного технического университета. 
UDK 621.314 .57

\title{
CONTROL DESIGN OF STANDALONE DISTRIBUTION GENERATION SYSTEM IN QUATERNION DOMAIN
}

Oleg V. Nos ${ }^{1}$,

nos@corp.nstu.ru

\author{
Aleksandr V. Korovin'2, \\ akvkorovin@ya.ru \\ Sergey V. Kuchak', \\ kuchak.2012@corp.nstu.ru \\ ${ }_{1}$ Novosibirsk State Technical University, \\ 20, Karl Marx avenue, Novosibirsk, 630073, Russia. \\ 2 AO PO «SEVER», \\ 3, Obedineniya street, Novosibirsk, 630027, Russia.
}

\begin{abstract}
Relevance. The present paper is devoted to the practical issues of ensuring uninterrupted and quality power supply for standalone industrial facilities and quick response of power supply systems that become increasingly important in the context of continuously growing portion of renewable generation, such as wind turbines, solar photovoltaic arrays, tidal power units, characterized by inconsistency and randomness of power generation periods. At same time, the load also has an unpredictable nature of consumption, which consists in unbalanced connection, abrupt power drops and surges, and the introduction of non-linear consumers, which affects the quality of the voltage in the power supply system.

The main aim. The paper presents a method to develop a quick-response control strategy for a standalone three-phase power supply system for mineral mining and processing facilities to ensure the generated balanced voltages with sinusoidal waveforms.

Objects: standalone power supply system; PWM four-leg full-bridge inverter, single-/three-phase load.

Methods: quaternion algebra, noncommutative multiplication rules for imaginary units, closed-loop control, cascade systems.

Results. The authors have developed basic concept covering the organization and implementation of a standalone AC generation system based on three-phase voltage quaternion decomposition into a balanced sinusoidal component and other components caused by amplitude-phase unbalance and nonlinear loads. The described continuous law provides a high speed of instantaneous compensation of distortions at abruptly variable load. Experimental studies were carried out on various kinds and configurations of loads: single-phase, three-phase symmetric, and also two-phase, one phase of which has a non-linear character of current consumption.
\end{abstract}

\section{Key words:}

Standalone power supply system, voltage source inverter, single-phase load, three-phase load, voltage quaternion.

\section{REFERENCES}

1. Vasil'kov O.S., Batueva D.E., Homyakov K.A., Palyanicin P.S. Aligning the load schedule of enterprises through the use of hybrid energy storage. Izvestiya MGTU «MAMI», 2020, no. 1, pp. 27-34. In Rus.

2. Fraser M.E., Manning C.D., Wells B.M. Transformerless fourwire PWM rectifier and its application in AC-DC-AC converters. IEE Proceedings - Electric Power Applications, 1995, vol. 142, no. 6, pp. 410-416.

3. Jahns M., Maldonado M.A. A new resonant link aircraft power generating system. IEEE Transactions on Aerospace and Electronics Systems, 1993, vol. 29, no. 1, pp. 206-214.

4. Vechiu I., Curea O., Camblong H. Transient operation of a fourleg inverter for autonomous applications with unbalanced load. IEEE Transactions on Power Electronics, 2010, vol. 25, no. 2, pp. 399-407.

5. Nos O.V., Brovanov S.V., Dybko M.A. Development of active filtering algorithms for higher harmonics in electrical power circuits. Optoelectronics, Instrumentation and Data Processing, 2016, vol. 52, no. 6, pp. 557-562.

6. Hsu P., Behnke M. A three-phase synchronous frame controller for unbalanced load. $29^{\text {th }}$ Annual IEEE Power Electronics Specialists Conference. Fukuoka, Japan, 1998. pp. 1369-1374.

7. Rokrok E., Hamedani M.E. Comprehensive control scheme for an inverter-based distributed generation unit. Iranian Journal of Science and Technology, Transaction B: Engineering, 2009, vol. 33, no. B6, pp. 477-490.

8. Aboelsaud R., Ibrahim A., Garganeev A.G. Voltage control of autonomous power supply systems based on PID controller under unbalanced and nonlinear load conditions. International Youth
Conference on Radio Electronics, Electrical and Power Engineering. Moscow, 2019. 6 p.

9. Aboelsaud R., Ibrahim A., Garganeev A.G. Review of three-phase inverters control for unbalanced load compensation. International Journal of Power Electronics and Drive System, 2019, vol. 10, no. 1, pp. $242-255$.

10. Prabhakar N., Mishra M. Dynamic hysteresis current control to minimize switching for three-phase four-leg VSI topology to compensate nonlinear load. IEEE Transactions on Power Electronics, 2010, vol. 25, no. 8, pp. 1935-1942.

11. Zheng L., Le D. Control of a three-phase four-wire inverter. IECON 2012 - 38th Annual Conference on IEEE Industrial Electronics Society. Montreal, QC, Canada, 2012. pp. 316-320.

12. Nasiri R., Radan A. Adaptive pole-placement control of 4-leg voltage-source inverters for standalone photovoltaic systems. Renewable Energy, 2011, vol. 36, no. 7, pp. 2032-2042.

13. Rivera M., Yaramasu V., Llor A., Rodriguez J., Wu B., Fadel M. Digital predictive current control of a three-phase four-leg inverter. IEEE Transactions on Industrial Electronics, 2013, vol. 60, no. 11, pp. 4903-4912.

14. Luis R., Silva J.F., Quadrado J.C. Output voltage quality evaluation of stand-alone four-leg inverters using linear and nonlinear controllers. Energies, 2017, vol. 10, no. 4, pp. 1-20.

15. Nos O.V., Korovin A.V., Nos N.I., Kucher E.S. Quaternion control of four-leg inverter for distribution system with harmonicproducing load. The $11^{\text {th }}$ International Conference on Electrical Power Drive Systems ICEPDS 2020. Saint Petersburg, Russia, 2020. $6 \mathrm{p}$.

16. Komeno A.S.F., Filho A.L.F., Ishihara J.Y., Brasil V.P. Threephase quaternion power in three-wire systems. $19^{\text {th }}$ International 
Conference on Renewable Energy and Power Quality Journal ICREPQ'21. Almeria, Spain, 2021. pp. 493-498.

17. Nos O.V. Control strategy of shunt active power filter based on an algebraic approach. The $16^{\text {th }}$ International Conference of Young Specialists on Micro/Nanotechnologies and Electron Devices EDM 2015. Erlagol, Altai, Russia, 2015. pp. 459-463.

18. Nos O.V., Dudin A., Petzoldt J. The instantaneous power quaternion of the three-phase electric circuit with linear load. The 17th International Conference of Young Specialists on Micro/Nanotechnologies and Electron Devices EDM 2016. Erlagol, Altai, Russia, 2016. pp. 526-531.

Information about the authors
19. Duesterhoeft W.C., Schulz M.W., Clarke E. Determination of instantaneous currents and voltages by means of alpha, beta, and zero components. Transactions of the American Institute of Electrical Engineers, 1951, vol. 70, no. 2, pp. 1248-1255.

20. Park R.H. Two-reaction theory of synchronous machines. Transactions of the American Institute of Electrical Engineers, 1929, vol. 48, no. 3, pp. 716-727.

Received: 29 October 2021.

Oleg V. Nos, Dr. Sc., professor, Novosibirsk State Technical University.

Aleksandr V. Korovin, deputy chief designer, AO PO «SEVER».

Sergey V. Kuchak, Cand. Sc., senior lecturer, Novosibirsk State Technical University. 